\section{A semiquantitative scoring tool to evaluate eccentric exercise- induced muscle damage in trained rats}

\author{
D. Rizo-Roca, ${ }^{1}$ J.G. Ríos-Kristjánsson, ${ }^{1}$ \\ C. Núñez-Espinosa, ${ }^{1}$ A.A. Ascensão, ${ }^{2}$ \\ J. Magalhães, ${ }^{2}$ J.R. Torrella, ${ }^{1}$ T. Pagès, ${ }^{1}$ \\ G. Viscor ${ }^{1}$
}

\section{'Department of Physiology and \\ Immunology, Faculty of Biology, \\ University of Barcelona, Spain \\ ${ }^{2}$ Research Centre in Physical Activity, Health and Leisure, Faculty of Sport, University of Porto, Portugal}

\section{Abstract}

Unaccustomed eccentric exercise is a welldocumented cause of exercise-induced muscle damage. However, in trained subjects muscle injury involves only light or moderate tissue damage. Since trained rats are widely used as a model for skeletal muscle injury, here we propose a semiquantitative scoring tool to evaluate muscle damage in trained rats. Twenty male Sprague-Dawley rats were trained for two weeks following a two-week preconditioning period, and randomly divided into two groups: control rats (CTL; $n=5)$ and rats with eccentric exercise-induced muscle damage (INJ; $\mathrm{n}=15$ ). Injured rats were sacrificed at three time points: 1,3 and 7 days post injury ( $n=5$ each). Transverse sections from the right soleus were cut $(10 \mu \mathrm{m})$ and stained with haematoxylineosin. Samples were evaluated by two groups of observers (four researchers experienced in skeletal muscle histopathology and four inexperienced) using the proposed tool, which consisted of six items organised in three domains: abnormal fibre morphology, necrotic/(re) degenerating fibres (muscle fibre domain), endomysial and perimysial infiltration (inflammatory state domain) and endomysium and perimysium distension (interstitial compartment domain). We observed the expected time course in the six evaluated items. Furthermore, agreement among observers was evaluated by measuring the Intraclass Correlation Coefficient (ICC). Within the experienced group, items from the muscle fibre and interstitial compartment domains showed good agreement and the two items from the infiltration compartment domain showed excellent agreement. In conclusion, the proposed tool allowed quick and correct evaluation of light to moderate muscle damage in trained rats with good agreement between observers.

\section{Introduction}

Several muscle injury models have been developed in order to better understand the underlying mechanisms involved in muscle damage assessment and recovery. These various models can be classified as either contraction-induced, resulting from eccentric contractions or strenuous exercise; or traumainduced, due to exposure of the muscle to a toxin, laceration or crush injury. ${ }^{1}$ Among the contraction-induced models, the eccentric exercise-induced muscle damage model is one of the most widely used in physiology laboratories. ${ }^{2,3}$ Unlike models of extreme physical trauma, in eccentric exercise-induced muscle damage only a relatively small proportion of fibres are affected, with most of the muscle cells remaining healthy and functional. ${ }^{4-7}$ Moreover, some training programmes can also reduce the amount of damage produced by eccentric exercise-induced muscle damage. It is well known that prior bouts of eccentric contractions provide a protective effect against eccentric exercise-induced muscle damage but, although numerous hypotheses have been proposed and tested, the unifying mechanism remains unclear. ${ }^{8,9}$ In terms of the effect of non-specifically eccentric training, such as level running, few studies have been conducted. In a study by Schwane and Armstrong, ${ }^{10}$ the level running group showed a certain degree of protection from further eccentric exerciseinduced damage. Another study found that, after downhill running, endurance-trained rats had lower serum creatine kinase activity than sedentary rats, ${ }^{11}$ while Koh and colleagues demonstrated that lengthening contractions are not necessary to induce protection from eccentric muscle damage. ${ }^{12}$ Some of the mechanisms responsible for the damage induced by eccentric exercise, such as loss of calcium homeostasis and the inflammatory response, ${ }^{13}$ are known to be regulated by endurance training. ${ }^{14,15}$ Moreover, endurance training can increase the slow oxidative fibre population through fibre-type conversion from fast to slow. ${ }^{16}$ Given that fast fibres are more susceptible to eccentric exercise-induced muscle damage than slow fibres, ${ }^{17}$ trained rats could be more protected against eccentric contraction due to their fibre type composition. Furthermore, in level running the soleus muscle (a predominantly slow muscle) also performs eccentric contractions during the gait cycle, probably developing some adaptations characteristic of specific eccentric training. ${ }^{18}$

Currently, in order to evaluate the impact of eccentric contraction-induced injuries, a wide variety of non-invasive functional and biochemical measurements are usually performed, including maximal voluntary contrac-
Correspondence: Dr. Joan Ramon Torrella, Department of Physiology and Immunology, Faculty of Biology, University of Barcelona, Av. Diagonal 643, 08005 Barcelona, Spain.

Tel. +34.934.039632 - Fax: +34.934 .110358 .

E-mail: jtorrella@ub.edu

Key words: Histopathology; haematoxylin-eosin; histochemical method.

Contributions: DRR, JRT, TP, GV, study conception and design; DRR, JGRK, CNE: care and training of experimental animals, data collection; DRR, JGRK, CNE, AA, JM, JRT, TP, GV: data analysis and interpretation; DRR, JRT, manuscript drafting DRR, JRT, JM, TP, GV, manuscript revision. Al authors read and approved the final manuscript.

Funding: this study was supported by DEP201022205-C02-01 and DEP2013-48334-C2-1-P grants (Spain's Ministry of Economy and Competitiveness) and MuscleTech Network project MTN20100101.

Received for publication: 15 June 2015. Accepted for publication: 1 October 2015

This work is licensed under a Creative Commons Attribution NonCommercial 3.0 License (CC BYNC 3.0).

(C) Copyright D. Rizo-Roca et al., 2015

Licensee PAGEPress, Italy

European Journal of Histochemistry 2015; 59:2544 doi:10.4081/ejh.2015.2544

tion torque and range of motion, swelling, ratings of soreness, maximum isometric force production, blood levels of myofibre protein and inflammatory cytokines and T2 signal intensity. ${ }^{19}$ In animal research (especially in rodent models) muscle biopsies and excisions are common, allowing histological approaches that can provide valuable information to better understand the injury phenomena. For the reasons mentioned above, moderate but appreciable damage is induced in endurance-trained subjects. Thus, we thought it necessary to develop an appropriate support tool to evaluate the muscle damage induced by eccentric exercise in trained rats, and here we present a sensitive histopathological semiquantitative tool that allows rapid evaluation of light to moderate muscle damage. At the histopathological level, features of muscle damage include fibre necrosis, swelling, an atrophic and sharp appearance, sarcomere and Z-line disruption, infiltration by phagocytic or inflammatory cells (neutrophils, macrophages, lymphocytes) interstitial oedema, enlarged interstitial area and extracellular matrix disruption. ${ }^{20}$ Since muscle damage is always followed by muscle regeneration (in healthy subjects), features of myofibre regeneration are also taken into 
account, including: small fibres within the intrafascicular area of a mature myofibre, round myofibres, central or internalised nuclei and basophilic cytoplasm. ${ }^{21,22}$ We used most of these well-known features to design a scoring method to evaluate muscle damage in the soleus muscle of trained rats, based on three major categories: histopathological abnormalities in the myofibres, the inflammatory state and interstitial compartment distension.

\section{Materials and Methods}

\section{Animals and experimental design}

Twenty male Sprague-Dawley run-trained rats were used for this study. All animals were maintained at an average temperature of $23^{\circ} \mathrm{C}$ under a light-dark cycle of $12 \mathrm{~h} / 12 \mathrm{~h}$ with food and water ad libitum. The animals were randomly divided into two experimental conditions: trained rats that did not suffer muscle injury before sampling (Control, CTL; $n=5$ ) and trained rats that were submitted to an eccentric exercise-induced muscle damage protocol and sacrificed 1, 3 or 7 days after muscle injury (Injured, INJ t01, t03 and t07 respectively; $\mathrm{n}=5$ each group).

All procedures were performed in accordance with the internal protocols of our laboratory, which were authorized by the University of Barcelona's Ethical Committee for Animal Experimentation and ratified, in accordance with current Spanish legislation, by the Departament de Medi Ambient $i$ Habitatge of the Catalan Government (Generalitat de Catalunya).

\section{Training protocol}

All animals were trained under environmental conditions $\left(21 \pm 2^{\circ} \mathrm{C}\right)$ on a flat treadmill (LE 8710; Panlab, Barcelona, Spain). After a twoweek preconditioning protocol in which the duration and intensity of the exercise were gradually increased, the training protocol began (Figure 1). This training period consisted of two daily running sessions for two weeks at a speed of $45 \mathrm{~cm} \mathrm{~s}^{-1}$ over $35 \mathrm{~min}$. According to Rodrigues et al., ${ }^{23}$ a rat running at a speed of $45 \mathrm{~cm} \mathrm{~s}^{-1}$ in a treadmill develops an exercise intensity of $90-100 \%$ of its $\mathrm{VO}_{2 \max }$. Due to the high intensity of exercise, it was scheduled to occur in two bouts with an interval of $6 \mathrm{~h}$ for recovery between sessions.

\section{Eccentric exercise-induced muscle damage protocol}

One, three or seven days after completion of the training period, skeletal muscle damage was induced in the INJ group by eccentric muscle contraction exercise, as described by Armstrong et al., ${ }^{3}$ consisting of downhill run- ning at $50 \mathrm{~cm} \mathrm{~s}^{-1}$ down an incline of $15^{\circ}$ until exhaustion. The protocol was applied twice on the same day: one session in the morning and one in the afternoon, with a rest period of $4 \mathrm{~h}$ between the end of the first session and the beginning of the second one.

\section{Muscle sampling}

Under animal's anaesthesia, the right soleus muscles were excised, immediately frozen in pre-cooled isopentane and then stored in liquid nitrogen. After dissecting out other tissues not involved in the present study, rats were killed by exsanguination.

\section{Light microscopic observations of histopathological changes}

Soleus muscles were placed in an OCT embedding medium (Tissue-Tek®; Sakura Alphen aan den Rijn, The Netherlands) at $22^{\circ} \mathrm{C}$ and serial transverse sections $(10 \mu \mathrm{m})$ of the equatorial region were cut using a cryostat (model CM3050S; Leica, Wetzlar, Germany) and stained with haematoxylin-eosin (HE) to evaluate the histopathological features of muscle damage. Stained samples were observed under a light microscope (BX61, Olympus, Tokyo, Japan) and photographed using a coupled camera (DP70, Olympus). To avoid sampling bias, and although the soleus muscle is homogeneous in fibre types, three pictures were taken from different areas and evaluated separately.

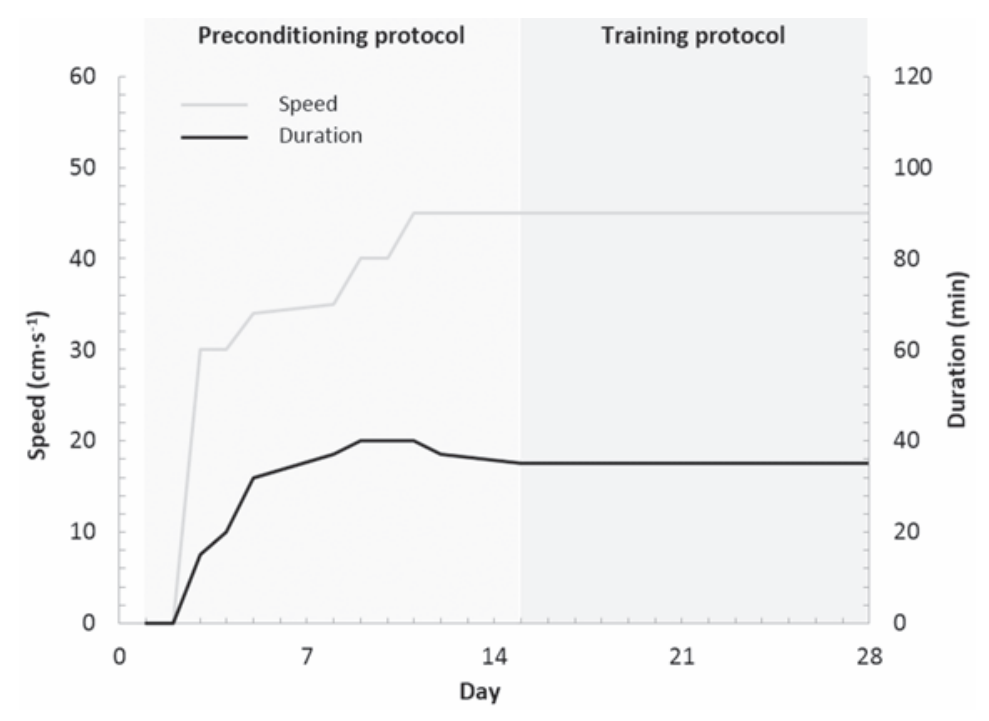

Figure 1. The exercise schedule for the preconditioning and training protocol, with two sessions per day, except for the first 4 days. During the first two sessions, the animals were placed on the treadmill, but did not run.

\section{Structure of the proposed semi- quantitative tool}

Three domains of histopathologic abnormality were chosen as the main elements of the scoring system: i) muscle fibre; ii) inflammatory state; and iii) interstitial compartment. Within each domain, and taking into account their importance, different items were determined considering their frequency of occurrence (items with a very low rate of occurrence were discarded) and physiological relevance (items strongly related to eccentric exerciseinduced muscle damage). The three domains were analysed independently to avoid bias in the relative importance of each one. All items were scored as 0,1 or 2 , as a wider range of scores would lead to a more confusing and slower tool. With just three values, which generically represent absence of damage (0), moderate damage (1) and severe damage (2), the classification of each item is more intuitive and faster without losing accuracy.

Three pictures for each sample each covering an area of $0.55 \mathrm{~mm}^{2}$ (approximately 120 fibres per picture) were analysed. Three measurements (one per picture) were taken for each item and subject, but only the highest score was considered. This criterion was applied to avoid score dilution. As stated above, muscle damage in trained rats is moderate and usually focused in a few fibres. Consequently, large areas of the samples remain undamaged and would receive a low score. If all the scores 
were considered, given enough pictures, even rats that showed great but focused damage would have obtained a score close to 0 .

Table 1 shows the chosen domains and items and their threshold for the different scores. These thresholds were established experimentally using CTL samples. An explanation for each domain and item is given in the following sections.

\section{Muscle fibre domain}

Healthy, uninjured muscle tissue exhibits relatively uniform polygonal myofibres that contain many peripherally placed nuclei. Intact sarcolemma and non-fragmented sarcoplasm are also characteristics of normal myofibres.

This domain was divided into two items based on their severity: i) Fibres with abnormal morphology (abnormal morphology item), which may or may not have retained their functionality. Abnormal fibre morphology included fibre atrophy (small, angulated or rounded fibres), swollen appearance or fibre splitting. ii) Severely damaged fibres that were undergoing necrosis or myophagocytosis (necrotic/(re)degenerating fibres item). Necrotic and degenerating fibres were identified by the presence of infiltrating inflammatory cells (myophagocytosis), fragmented sarcoplasm, dark staining (hypercontracted fibres) and pale staining (necrotic fibres), while regenerating fibres were, in the early stages, represented by small basophilic myotubes and later by bluish-stained myofibres with central nuclei. ${ }^{24}$

Given that haematoxylin and eosin was the only staining protocol used, differentiating between necrotic and regenerating fibres was sometimes difficult. Additionally, regenerative processes are usually preceded by necrosis. For these reasons, necrotic, degenerating and regenerative fibres were included as a single item.

\section{Extracellular inflammatory domain}

The inflammatory response is well studied in eccentric exercise-induced muscle damage and is characterized, besides several biochemical-related consequences, by leukocyte infiltration. ${ }^{25}$ Although neutrophils, macrophages and lymphocytes are involved in this response, their differentiation would be time and resource consuming, as several antibodies would be needed in order to identify each cell type. Consequently, the inflammatory domain took into account all leukocytes together, but was divided into two items according to their localization: i) perimysial infiltration; and ii) endomysial infiltration.

\section{Interstitial compartment}

In physiological conditions, muscle fibres are tightly packed together into fascicles sepa- rated by scant connective tissue. The extracellular matrix is a very dynamic compartment and responds to mechanical stress, ${ }^{26}$ and can be disrupted as a consequence of the eccentric stimulus, leading to an inflammatory response $^{27}$ and fibrotic process. Therefore, we evaluated the distension of the interstitial compartment, divided into the items i) endomysium; and ii) perimysium.

\section{Statistical methods}

All samples were evaluated by two groups of observers: the first group comprised four researchers experienced in skeletal muscle histology, and the second group comprised four researchers who were inexperienced in this field of study. In order to assess the degree of agreement among observers, the Intraclass
Correlation Coefficient (ICC) was calculated using MedCalc for Windows ver. 15 (MedCalc Software, Ostend, Belgium). This measure is used to demonstrate consensus and agreement among observational ratings provided by multiple observers or instruments, 1 being the maximum value for an ICC measure. ${ }^{28}$ In our study, the data was modelled assuming that the same observers would evaluate all samples, although these observers may be a subset of a larger set of observers. ICC values are presented with a $95 \%$ confidence interval $(95 \% \mathrm{CI})$. The level of agreement can be interpreted as follows: $<0.40=$ poor; $0.40-0.59=$ fair; 0.60 $0.74=$ good; and $\geq 0.75=$ excellent. These cut offs, although arbitrary, are commonly used. ${ }^{29}$

Histological scores were compared using a Kruskal-Wallis test followed by Dunn's multiple

Table 1. Structure of the scoring tool used to evaluate eccentric exercise-induced muscle damage.

\begin{tabular}{lcc}
\hline Domains and items & Score & Definition \\
Muscle fibre & & \\
Abnormal morphology & 0 & 4 fibres \\
Small, rounded or angular fibres, splitting & 1 & 4 to 7 fibres \\
and hypertrophied fibres were considered abnormal & 2 & $>7$ fibres or an entire fascicle \\
Necrotic/(re)degenerating & 0 & Absent \\
Basophilic, light stained, central nuclei & 1 & 1 to 2 fibres \\
and myophagocytosed fibres & 2 & $>2$ fibres \\
Extracellular inflammatory state & & \\
Endomysial infiltration & 0 & $<6$ cells \\
Small, mononuclear cells found in the endomysium & 1 & one cluster or $\geq 6$ cells \\
& 2 & $>1$ cluster or an entire fascicle infiltrated \\
Perimysial infiltration & 0 & $\leq 10$ cells \\
Small, mononuclear cells found in the perimysium & 1 & $>10$ cells \\
& 2 & $>2$ clusters or widely diffused \\
\hline Interstitial compartment & & Tight space \\
Endomysium distension & 0 & Moderately distended \\
Space between individual muscle fibres & 1 & Completely distended \\
Perimysium distension & 2 & Tight space \\
Space between fascicles & 0 & Moderately distended \\
& 1 & Completely distended \\
\hline
\end{tabular}

Table 2 . Intra-class coefficients with $95 \%$ confidence intervals evaluated by two groups of researchers ( $n=4$ each group).

\begin{tabular}{llcc} 
Domain & Item & \multicolumn{2}{c}{ ICC (95\% CI) } \\
Muscle fibre & Abnormal morphology & $0.71(0.55-0.85)$ & $0.38(0.20-0.63)$ \\
& Necrotic/(re)degenerating & $0.63(0.44-0.79)$ & $0.41(0.17-0.66)$ \\
Extracellular inflammatory state & Endomysial infiltration & $0.82(0.70-0.91)$ & $0.52(0.28-0.73)$ \\
& Perimysial infiltration & $0.78(0.65-0.89)$ & $0.49(0.23-0.72)$ \\
\hline Interstitial compartment & Endomysium distension & $0.69(0.52-0.83)$ & $0.28(0.02-0.56)$ \\
& Perimysium distension & $0.67(0.49-0.82)$ & $0.37(0.10-0.66)$ \\
\hline
\end{tabular}

ICC, Intra-class coefficients; Cl, confidence interval. 
comparison post hoc test. Statistical significance was considered as $\mathrm{P}<0.05$.

\section{Results}

\section{Application of the proposed semi- quantitative tool}

Soleus cross-sections from all groups were evaluated using the proposed tool (Table 1). As expected, the CTL group received the lowest score for each item while INJ t01 and t03 received the highest (Figure 2). When compared to CTL rats, the INJ t01 group had a significantly higher score for the following items: abnormal morphology $(\mathrm{P}=0.0188)$; necrotic and (re)degenerating fibres $(\mathrm{P}=0.0082)$, endomysial and perimysial infiltration ( $\mathrm{P}=0.0087$ and $\mathrm{P}=0.0049$, respectively), and perimysium distension $(\mathrm{P}=0.0343)$. INJ $\mathrm{t} 03$ rats followed a similar pattern, with significantly higher scores in abnormal morphology $(\mathrm{P}=0.0048)$, necrotic and (re)degenerating fibres $(\mathrm{P}=0.0382)$, and endomysial infiltration
$(\mathrm{P}=0.0029)$. Finally, INJ t07 did not differ significantly from the other groups, receiving for each item a mean score between the CTRL and INJ t01/t03 groups. Figure 3 shows representative pictures of each group.

\section{Agreement among observers on scoring data}

Six items were separately evaluated by four experienced and four inexperienced researchers in skeletal muscle physiology and morphology. In the experienced group, abnormal morphology, necrotic/(re)degenerating fibres, and endomysium and perimysium distension items showed Good agreement (ICC $\geq 0.60$ ), while the two items regarding infiltration (endomysial and perimysial infiltration) showed excellent agreement (ICC $\geq 0.75$ ). On the other hand, the group of four inexperienced researchers only showed poor and fair agreement (abnormal fibres and interstitial compartment items; and necrotic/(re)degenerating fibres and extracellular inflammatory state items, respectively). ICC values for each item are shown in Table 2.
Representative examples of the use of the proposed tool by the two groups of observers are shown in Figure 4.

\section{Discussion}

We developed the proposed tool in order to quickly and simply evaluate and understand the amount of muscle damage in trained rats. Although there are already numerous related semiquantitative measurement tools, none of them is sufficiently sensitive to detect/evaluate light to moderate damage. For example, Takekura et $a l .^{30}$ used a model in non-trained rats to evaluate different features, such as necrotic and spreading fibres, scoring from (no changes observed) to ++++ (up to $8 \%$ of fibres affected). Their lowest value for damage (score +) was up to $2 \%$. In contrast, in our trained rats this value would represent an important amount of damage (score 2).

Other methods use the percentage of injured fibres to evaluate muscle damage, for
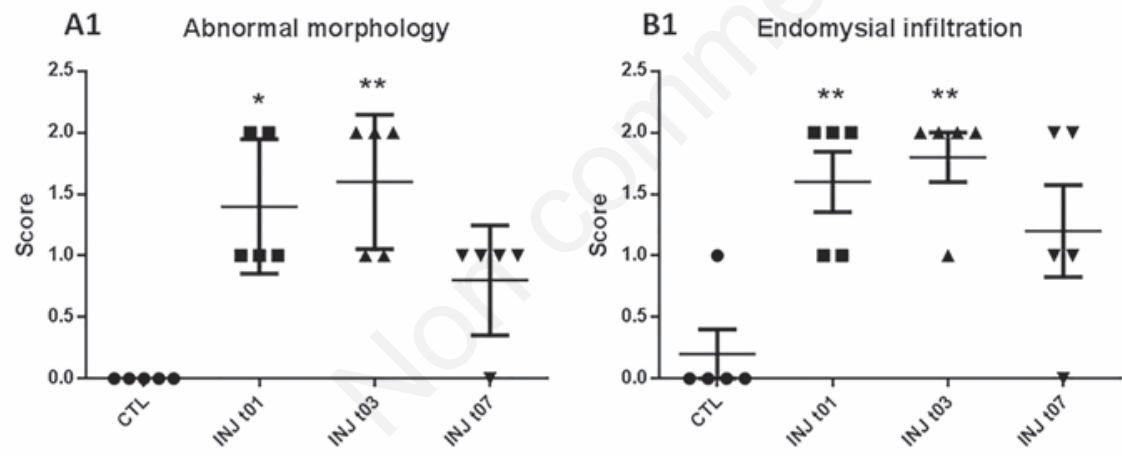

A2 Necrotic/(Re)Degenerating fibres

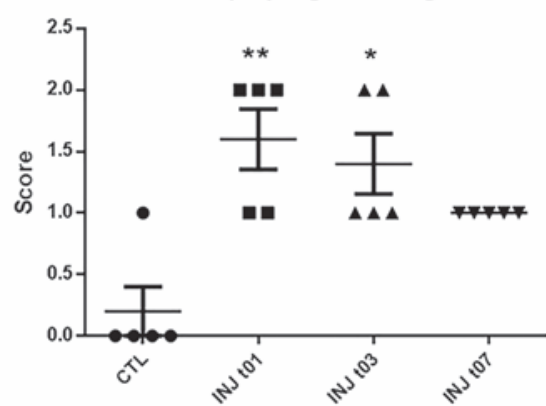

B2

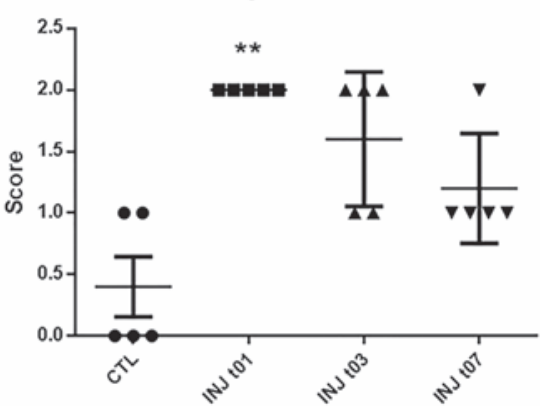

C1 Endomysium distension

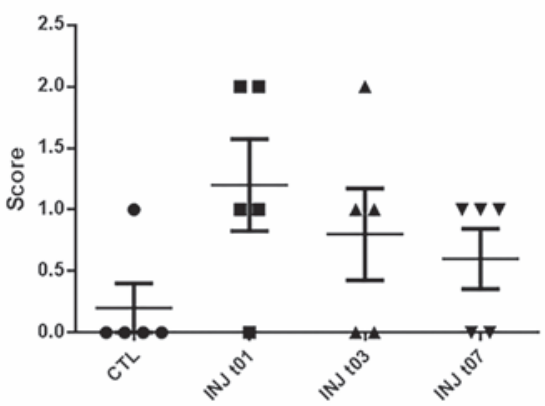

C2

Perimysium distension

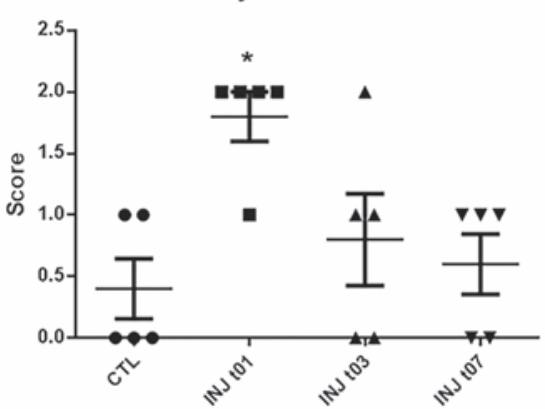

Figure 2. Scatter plot in which each symbol (dots, squares and triangles) represents the given score for a subject. Mean and SEM are represented. Asterisks (one or two) represent significant differences vs CTL $(\mathrm{P}<0.05$ and $\mathrm{P}<0.01$, respectively). A1 and A2, muscle fibre domain items; B1 and B2, extracellular inflammatory domain items; C1 and C2, interstitial compartment items. 
example, McCormack et al.$^{31}$ used this method when evaluating muscle injury in a model of ischaemia/reperfusion, Koh et al. ${ }^{12}$ applied it after stimulating the peroneal nerve to evaluate the damage produced in extensor digitorum longus by repeated lengthening contractions. However, given the small number of injured fibres present in trained animals, evaluating injury based on the percentage of dam- aged fibres will not provide reliable results. Wedderburn et al. ${ }^{32}$ used a scoring system involving four domains and 16 items (contrasting with our three domains and six items), which represents a more exhaustive way of evaluating the damage. Their tool is adequate for clinical and diagnostic purposes but is highly time-consuming, contrasting with the rapidity of our method, when applied to a model of trained animals.

Therefore, we here propose a semiquantitative approach that offers a compromise between reliability and speed, and allows samples to be classified in whole numbers as scores $(0,1$ or 2$)$. The proposed tool was found to be able to discriminate between healthy and damaged samples. For all items, the CTL group received a mean score below 1 , significantly
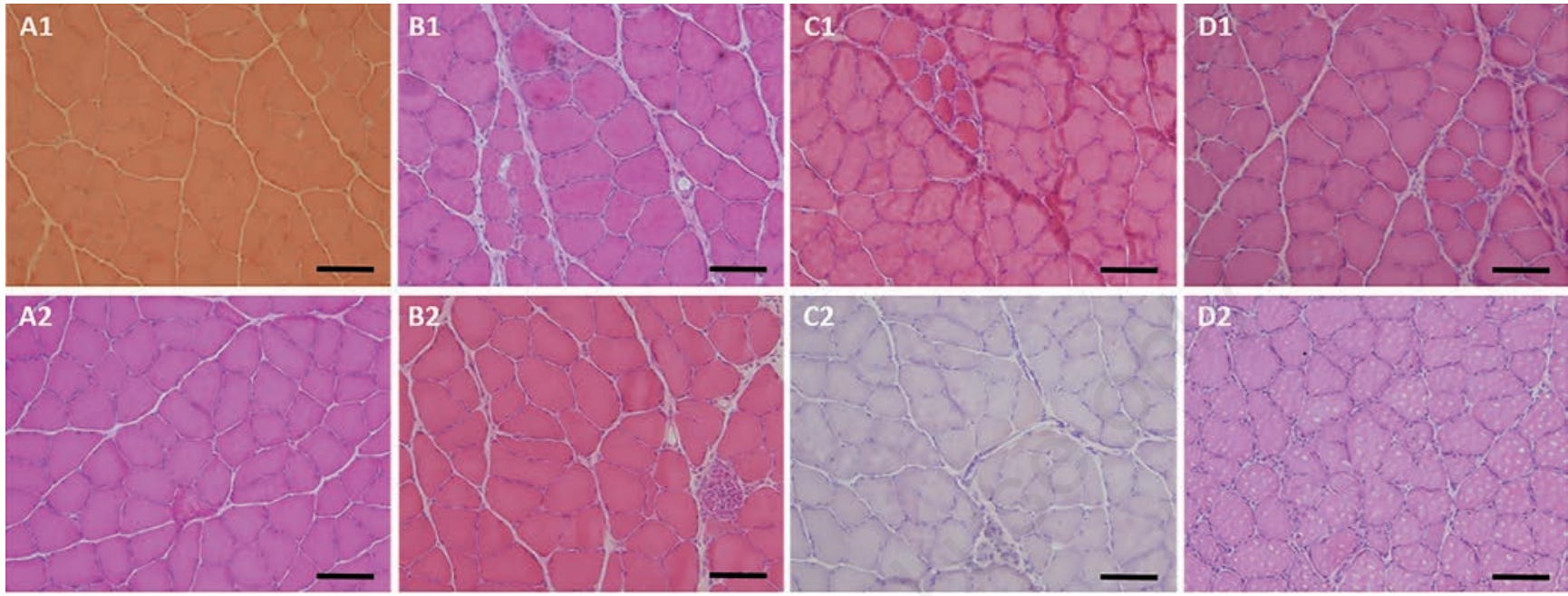

Figure 3. Representative microphotographs of CTL rats (A1 and A2), INJ t01 (B1 and B2), INJ t03 (C1 and C2) and INJ t07 (D1 and D2). Degeneration and myophagocytosis appear in pictures B2, C1 and C2. Endomysial infiltration is evident in B1, C1 and D1. Scale bars: $100 \mu \mathrm{m}$.

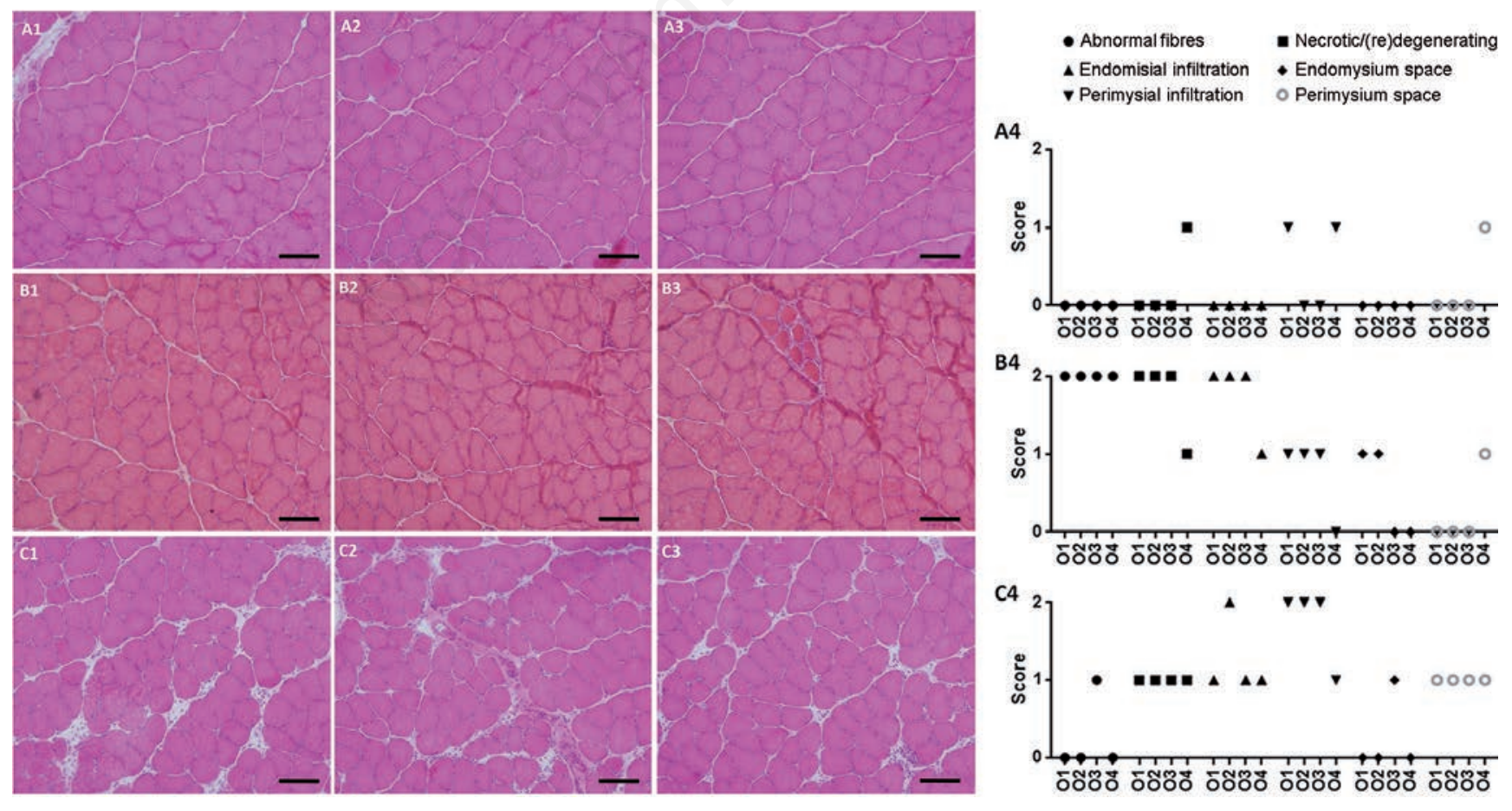

Figure 4. Microphotographs of evaluated pictures. A4, B4 and C4 show the respective scores given by four experienced observers (O1O4). Only the highest score from each picture was ultimately taken into account in order to better represent great anomalies such as those found in B3. Scale bars: $100 \mu \mathrm{m}$. 
lower than the scores received by the INJ t01 group (except for the Endomysium distension item). Furthermore, for all items, the tool showed the time course described in the literature following exercise-induced muscle damage: an early acute inflammatory and degenerative phase (highest scores are in the $\mathrm{t} 01$ and $\mathrm{t} 03$ groups) followed by a later stage ( $\mathrm{t} 07$ ) with diminished signs of degeneration and inflammation.

Regarding the interstitial compartment domain, both endomysium and perimysium distension items showed the same time course as the previous domains, although without statistical differences when the different time points were compared, with the exception of Perimysium distension in the INJ t01 group. There was much interindividual variance among subjects, with each of the three possible scores co-existing within each group. Thus, although the trend was clear, there were no significant differences.

\section{Inter-rater reliability}

A representative example of scoring using the proposed semiquantitative tool (Table 1) is shown in Figure 4. Good agreement between the four observers was apparent in most items.

Regarding the degree of agreement among the four observers, the items belonging to the inflammatory state domain (endomysial and perimysial infiltration) obtained the highest ICC (Table 2), which qualified as excellent. The fact that mononuclear infiltrating cells are easily identifiable, as well as the easily distinguishable features of score 0 and 2 (Table 1), are key factors explaining this high agreement. The lowest ICC (0.63), which still qualified as good, was obtained for the item necrotic/(re)degeneration. This could be explained by the fact that some of its features are slightly subjective. For example, cytoplasm coloration, namely basophilic or pale staining, is an important marker used to classify a fibre for this item, but its evaluation depends, to some degree, on observer sensitivity. With regards to the Abnormal fibres item, good agreement (ICC $=0.71$, close to the excellent threshold) was obtained. The interstitial compartment domain (endomysium and perimysium distension), despite being purely qualitative and subjective (these items were evaluated as not distended, moderately distended and completely distended) also showed Good agreement (ICC $=0.69$ and 0.67). Although the ICC is a reliable statistical test for assessing agreement, ${ }^{30}$ we decided to add an additional group of observers to definitively rule out the possibility that the consensus occurred by chance. This additional group of four observers comprised researchers who were inexperienced in skeletal muscle histopathology. Effectively, this group demonstrated poor and fair levels of agreement for all items.

In conclusion, the proposed semiquantitative scoring tool offers a quick, simple, sensitive and understandable method for evaluating eccentric exercise-induced muscle damage in trained rats by using a simple, widely used and highly standardised procedure such as haematoxylin-eosin staining. The scoring system showed the expected time course of muscle injury when tested on samples of trained rats. Moreover, it generated enough agreement to be generally used by researchers who need to assess light to moderate muscle damage in rats. Potential limitations must be taken into account: application of the tool is restricted to research purposes, and the threshold between scores should be empirically adjusted depending on the model and degree of muscle damage.

\section{References}

1. Warren GL, Palubinskas LE. Human and animal experimental muscle injury models. In: Tiidus PM (ed.) Skeletal muscle damage and repair. Human Kinetics, Champaign; 2007.

2. Souza J De, Gottfried C. Muscle injury: review of experimental models. J Electromyogr Kinesiol 2013;23:1253-60.

3. Armstrong RB, Ogilvie RW, Schwane JA. Eccentric exercise-induced injury to rat skeletal muscle. J Appl Physiol 1983;54:8093.

4. Carter GT, Kikuchi N, Abresch RT, Walsh SA, Horasek SJ, Fowler WM. Effects of exhaustive concentric and eccentric exercise on murine skeletal muscle. Arch Phys Med Rehabil 1994;75:555-9.

5. Duan C, Delp MD, Hayes DA, Delp PD, Armstrong RB. Rat skeletal muscle mitochondrial $[\mathrm{Ca} 2+]$ and injury from downhill walking. J Appl Physiol 1990;68:1241-51.

6. Komulainen J, Vihko V. Exercise-induced necrotic muscle damage and enzyme release in the four days following prolonged submaximal running in rats. Pflugers Arch 1994;428:346-51.

7. Ogilvie RW, Armstrong RB, Baird KE, Bottoms CL. Lesions in the rat soleus muscle following eccentrically biased exercise. Am J Anat 1988;182:335-46.

8. Howatson G, van Someren KA. The prevention and treatment of exercise-induced muscle damage. Sports Med 2008;38:483-503.

9. McHugh MP. Recent advances in the understanding of the repeated bout effect: the protective effect against muscle damage from a single bout of eccentric exercise. Scand $\mathbf{J}$ Med Sci Sports 2003;13:88-97.

10. Schwane JA, Armstrong RB. Effect of train- ing on skeletal muscle injury from downhill running in rats. J Appl Physiol 1983;55: 969-75.

11. Isanejad A, Saraf ZH, Mahdavi M, Gharakhanlou R, Shamsi MM, Paulsen G. The effect of endurance training and downhill running on the expression of IL1 , IL-6, and TNF- and HSP72 in rat skeletal muscle. Cytokine 2015;73:302-8.

12. Koh TJ, Brooks SV. Lengthening contractions are not required to induce protection from contraction-induced muscle injury. Am J Physiol Regul Integr Comp Physiol 2007;155-61.

13. Clarkson PM, Sayers SP. Etiology of exercise-induced muscle damage. Can J Appl Physiol 1999;24:234-48.

14. Inashima S, Matsunaga S, Yasuda T, Wada M. Effect of endurance training and acute exercise on sarcoplasmic reticulum function in rat fast- and slow-twitch skeletal muscles. Eur J Appl Physiol 2003;89:142-9.

15. Koh TJ. Do small heat shock proteins protect skeletal muscle from injury? Exerc Sport Sci Rev. 2002;30:117-21.

16. Pette D, Staron RS. Mammalian skeletal muscle fiber type transitions. Int Rev Cytol 1997;170:143-223

17. Macpherson PC, Schork MA, Faulkner JA. Contraction-induced injury to single fiber segments from fast and slow muscles of rats by single stretches. Am J Physiol Cell Physiol 1996;271:C1438-46.

18. Dugan S a, Bhat KP. Biomechanics and analysis of running gait. Phys Med Rehabil Clin N Am 2005;16:603-21.

19. Warren GL, Lowe DA, Armstrong RB. Measurement tools used in the study of eccentric contraction-induced injury. Sports Med 1999;27:43-59.

20. Sayers SP, Hubal MJ. Histological, Chemical and functional manifestations of muscle damage. In: Tiidus PM (ed.) Skeletal muscle damage and repair. Human Kinetics, Champaign; 2007.

21. Ciciliot S, Schiaffino S. Regeneration of mammalian skeletal muscle: basic mechanisms and clinical implications. Curr Pharm Des 2010;16:906-14.

22. Sudo M, Kano Y. Myofiber apoptosis occurs in the inflammation and regeneration phase following eccentric contractions in rats. J Physiol Sci. 2009;59:405-12.

23. Rodrigues B, Figueroa DM, Mostarda CT, Heeren MV, Irigoyen MC, De Angelis K. Maximal exercise test is a useful method for physical capacity and oxygen consumption determination in streptozotocin-diabetic rats. Cardiovasc Diabetol 2007;6:38.

24. Sewry C, Goebel HH. General pathology of muscle disease. In: Bönnemann C (ed.) Muscle disease. J. Wiley \& Sons, Oxford; 2013. 
25. Peake J, Nosaka K, Suzuki K. Characterization of inflammatory responses to eccentric exercise in humans. Exerc Immunol Rev 2005;11:64-85.

26. Kovanen V. Intramuscular extracellular matrix: complex environment of muscle cells. Exerc Sport Sci Rev 2002;30:20-5.

27. Stauber WT, Clarkson PM, Fritz VK, Evans WJ. Extracellular matrix disruption and pain after eccentric muscle action. J Appl Physiol 1990;69:868-74.

28. Shrout PE, Fleiss JL. Intraclass correla- tions: uses in assessing rater reliability. Psychol Bull 1979;86:420-8.

29. Cicchetti D.V. Guidelines, criteria, and rules of thumb for evaluating normed and standardized assessment instruments in psychology. Psychol Assess 1994;6:284-90

30. Takekura H, Fujinami N, Nishizawa T, Ogasawara H, Kasuga N. Eccentric exercise-induced morphological changes in the membrane systems involved in excitation-contraction coupling in rat skeletal muscle. J Physiol 2001;533:571-83.
31. McCormack MC, Kwon E, Eberlin KR, Randolph M, Friend DS, Thomas AC, et al. Development of reproducible histologic injury severity scores: skeletal muscle reperfusion injury. Surgery 2008;143:126-33.

32. Wedderburn LR, Varsani H, Li CKC, Newton KR, Amato A, Banwell B, et al. International consensus on a proposed score system for muscle biopsy evaluation in patients with juvenile dermatomyositis: a tool for potential use in clinical trials. Arthritis Rheum 2007;57:1192-201. 